\title{
Blocking and appetitive reinforcement
}

\author{
EDWARD S. HALAS and MARILOU J. EBERHARDT \\ Grand Forks Human Nutrition Research Center, Grand Forks, North Dakota
}

\begin{abstract}
Thirty-six male and female rats were trained and tested in a blocking paradigm using appetitive reinforcement. For half of the animals, light was used as the original stimulus, whereas a tone was used as the original stimulus for the remaining half. In Stage 1, the original stimulus was reinforced with a food pellet when the rat pressed the lever. Leverpresses were not reinforced when the stimulus was off. In Stage 2, a redundant stimulus was paired with the original stimulus and reinforced with a pellet for each leverpress. In Stage 3, the rats were tested for blocking. Light and tone were equally effective in blocking the redundant stimulus. Both males and females exhibited blocking. These results are in agreement with those of studies using aversive reinforcement.
\end{abstract}

Kamin's original (1969) study was the impetus for a renewed effort to investigate the variables affecting Pavlovian conditioning. The resulting theoretical debates and diversity of research were perhaps unexpected but very rewarding. Interestingly, this very energetic field of research has relied mainly on the conditioned emotional response and aversive reinforcement as tools for studying these variables. Very little research has been done using appetitive reinforcement, although Kamin stated that appetitive reinforcement should yield the same results as aversive reinforcement.

Some studies have utilized appetitive reinforcement in blocking experiments, but the methodologies used were not well suited to illustrate the sequential development of the blocking phenomenon. For instance, Holland (1977) was primarily interested in determining the unique types of conditioned responses (CRs) that were elicited by tone and light conditioned stimuli (CSs). He found that the nonpreferred stimulus (e.g., light) could block the development of a CR (head jerk) when the preferred stimulus (tone) was added in a compound conditioning procedure. Neely and Wagner (1974), on the other hand, were primarily interested in the attenuation of blocking due to different schedules of reinforcement. Because of the type of methodology used in their study, they concluded that it was not possible to assess the magnitude of acquisition to element A prior to the compound conditioning of AX. They felt this lack of data prevented a proper evaluation of the Rescorla and Wagner (1972) model. In Dickinson and Mackintosh's (1979) study, the food reinforcement was delivered at the termination of the 30-sec CS. Any responses made during the CS period were not rewarded. Consequently, the performance curves in their Experiment 2 were essentially flat across the 6 days of training in Stages 1 and 2. Conditioning was not obvious until the rats were tested for their response to the redundant stimu-

Address correspondence and reprint requests to Edward S. Halas, United States Department of Agriculture, Agricultural Research Service, Grand Forks Human Nutrition Research Center, P. O. Box 7166, University Station, Grand Forks, ND 58202. lus. In most studies, as the animal is conditioned, there is a progressive change in the performance curves.

The purpose of the present study was to demonstrate blocking in a conventional appetitive paradigm. In Kamin's (1969) study, rats were trained on a variable-interval (VI) schedule to obtain food pellets. The daily sessions lasted $2 \mathrm{~h}$. A 3-min stimulus (light or noise) was presented four times during the training session. At the termination of the stimulus, the rats received an electric shock. The rats quickly learned to suppress their responses during the stimulus period. In the second stage of the experiment, a redundant stimulus was paired with the original stimulus and training continued, with the compound stimuli reinforced with the shock. In the third stage, the redundant stimulus was tested for its ability to suppress leverpressing. A suppression ratio was computed and statistically analyzed. In the present study, these procedures were modified. Food pellets were obtained only during the stimulus period and no shocks were given. Because the pellets could be earned only while the stimulus was on, the stimulus was presented 15 times during the 2-h training session. The stimulus duration was $2 \mathrm{~min}$.

\section{METHOD}

\section{Subjects}

Thirty-six hooded Long-Evans rats, 18 males and 18 females, were used. The females were 140 days old; the males were 150 days old. All animals were housed in individual stainless steel cages with water bottles.

\footnotetext{
Apparatus

Nine Scientific Products ${ }^{1}$ operant boxes were individually housed in sound-attenuated chambers. The inside dimensions of each chamber were $39 \mathrm{~cm}$ (depth) $\times 48 \mathrm{~cm}$ (height) $\times 54 \mathrm{~cm}$ (width). The four walls, ceiling, and floor were constructed of 5-cm thick Styrofoam. A blower fan forced air into each chamber and also provided background noise. An S-shaped exhaust pipe, painted flat black inside and outside, was installed in each chamber to allow free air flow and to prevent any light from entering the chamber. Each operant box measured $20 \mathrm{~cm} \times 21 \mathrm{~cm}$ $\times 24 \mathrm{~cm}$. Two panels of the operant box were made of clear Plexiglas; the two end panels were metal. A nonretractable lever, food cup, and 4-W white plastic-covered light were mounted on one of the metal panels. A Gerbrands dispenser fed 45-mg pellets into the food cup. A Sonalert
} 
was mounted in the center of the ceiling panel of the operant box. An IBM personal computer (PC) controlled the onset/offset of the light, Sonalert, and pellet dispenser. The PC also collected the data from each animal.

\section{Procedure}

All rats were reduced to $80 \%$ of their normal body weights and were handled for $5 \mathrm{~min}$ daily for 5 days prior to the start of the experiment. During 1 of the handling days, the rats were put in the operant boxes in the afternoon and were reinforced on a continuous reinforcement (CRF) schedule until the next morning, when they were returned to their home cages. The rats were given just the one overnight CRF session in the operant boxes. All rats were then trained to an original stimulus, either light (L) or tone (T), for 5 days during Stage 1, followed by 4 days of training to paired light/tone (LT) in Stage 2. The 18 females were run first. Nine females were trained for 5 days to the $L$ stimulus in Stage 1, while the second group of 9 females was trained for 5 days to the $\mathrm{T}$ stimulus in Stage 1 .

For the $\mathrm{L}$ group, over the 2-h session, the light mounted on the wall panel was turned on 15 times for 2 min each time. The light generated $1 \mathrm{fc}$, as measured by a Simpson Model 408-2 illumination level meter. The interstimulus intervals varied randomly from 3 to $7 \mathrm{~min}$. Any leverpress that occurred while the light was on was rewarded with a food pellet. No pellets were given for leverpresses when the light was off. The number of leverpresses that occurred $2 \mathrm{~min}$ prior to the stimulus (prestimulus period) and the number of leverpresses that occurred during the stimulus period were recorded for each rat by the PC. At the end of the 2-h training session, the number of leverpresses for the 15 prestimulus periods and the 15 stimulus periods were totaled and a ratio (stimulus period/[prestimulus period + stimulus period]) was computed for each rat. Like Kamin's (1969) suppression ratio, a ratio of 0.00 would signify no leverpressing during the stimulus period, whereas a ratio of 0.50 would indicate an equivalent number of leverpresses during the prestimulus and stimulus periods. A ratio approaching 0.99 would signify a high rate of leverpressing during the stimulus period and very little leverpressing during the prestimulus period. At the completion of the 2-h training session, the rats were weighed, returned to their home cages, and given sufficient food to maintain their $80 \%$ body weights.

The same procedure was used to run the second group of females, except that a tone was used as the stimulus. The daily 2-h training sessions for each group were given at the same time each day. The tone was generated by the Sonalert mounted in the ceiling of the operant box. The frequency of the Sonalert was $2900 \mathrm{~Hz}$ at an intensity of $93 \mathrm{~dB}$, as measured inside the operant box. The 93-dB level for the tone was set while the blower fan was not operating. The background noise generated by the blower fan was $56 \mathrm{~dB}$. The decibel levels for the tone and background noise were measured by a Simpson Model 886 Type 2 Band A sound level meter. The tone consisted of discrete beeps. During the 2-min stimulus period, the tone was on for $180 \mathrm{msec}$ and off for $340 \mathrm{msec}$.

Twenty-four hours after the completion of Stage 1, both groups of females were given 4 days of training on the paired LT stimulus in Stage 2. The light and tone used in Stage 1 were used in Stage 2.

Twenty-four hours after the completion of Stage 2, the $L$ rats were tested for blocking in Stage 3, using a L-T-T-L sequence. The interstimulus intervals varied randomly from 3 to $7 \mathrm{~min}$. The L-T-T-L sequence was repeated four times during the 2-h testing session. No food pellets were given at any time in Stage 3 . The number of leverpresses while the $\mathrm{L}$ and $\mathrm{T}$ stimuli were on was recorded by the PC. To measure the magnitude of blocking that occurred during the L-T-T-L sequence, the total number of leverpresses that occurred during the $T$ stimulus periods was divided by the total number of leverpresses recorded during the entire L-T-T-L sequence. The prestimulus periods were not used in computing this ratio. A ratio approaching 0.00 would indicate a high level of blocking, whereas a ratio of 0.50 would suggest no blocking. One ratio was computed for each of the four L-T-T-L sequences for each rat. A group mean ratio was then computed for each of the four sequences. To determine whether the group mean ratios were significantly different from $0.50, t$ tests were performed. If the $\mathrm{L}$ stimulus blocked conditioning to the $\mathrm{T}$ stimulus, it would be expected that a high number of leverpresses would be recorded during the two $L$ periods and few or no leverpresses would be recorded during the two $T$ periods. The same Stage 3 procedure was used for the rats trained on the T stimulus in Stage 1, except that for this group the sequence was T-L-L-T and the ratio was computed by dividing the total number of leverpresses during the $L$ stimulus periods by the total number of leverpresses during the entire $T$ - $L-L-T$ sequence.

The same procedures were used to train and test the 18 males.

An ANOVA was used to analyze the performance of the groups across days and differences among the groups (Kolstoe, 1973). The $t$ test was used to analyze differences between the group mean ratio and $\mathbf{0 . 5 0}$.

\section{RESULTS}

All four groups, as is apparent in Figure 1, improved their performance $(p<.0002)$ across the 5 days of training in Stage 1. When the data for the $\mathrm{T}$ and $\mathrm{L}$ stimuli were analyzed for their rate of conditioning, no significant differences $(p>.05)$ were found. Thus, the two stimuli were equally salient. When the data were analyzed by sex, however, the performance of the males was found to be superior to that of the females $[F(1,168)=20.93$, $p<.0001]$. After 5 days of training in Stage 1, all of the groups had reached their performance asymptote. There was no further improvement in performance in Stage 2 for any of the groups $(p>.05)$. In Stage 2, there were again no differences $(p>.05)$ between the $\mathrm{L}$ and T stimuli, but there was a sex difference $[F(1,135)=$ $9.37, p<.003]$. The males were superior in performance to the females. Blocking was observed in Stage 3 to both $\mathrm{L}$ and $\mathrm{T}$ for both males and females. For both males and females in the $\mathrm{L}$ group, blocking was observed in $\mathrm{Se}$ quences $1(p<.008)$ and $4(p<.02)$, but not in Sequences 2 and $3(p>.05)$. The female T group did not exhibit blocking in Sequence $2(p>.05)$, although the male $\mathrm{T}$ group exhibited blocking in all sequences. We

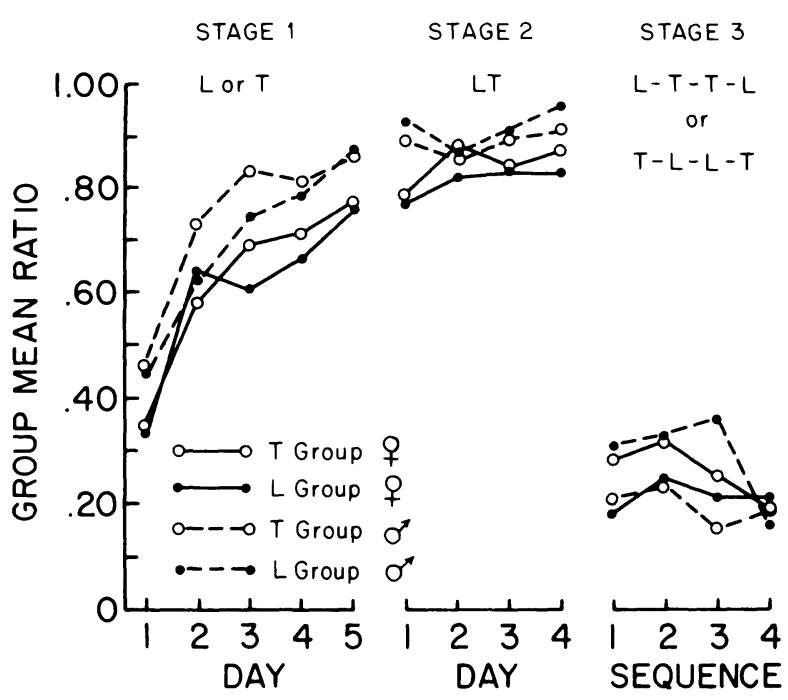

Figure 1. In Stage 1, 9 female and 9 male rats were trained to light (L) for 15 trials per day for 5 days. Nine female and 9 male rats were trained to tone (T) during the same time period. In Stage 2 , all rats were trained to the paired LT for 15 trials per day for 4 days. In Stage 3, the rats were tested for blocking. Both $L$ and $T$, as original stimuli in Stage 1 , were able to block the redundant stimulus in Stage 2. 
have no explanation for these findings, except that with only 9 rats per group, a few deviant rats could impact markedly on group mean and variability. In the male $\mathrm{L}$ group, 3 of the 9 rats did not show blocking.

Significant extinction to both $\mathrm{L}$ and $\mathrm{T}$ was exhibited by both males and females. In Sequence 1, the females averaged 44 leverpresses to the original stimulus and 19 to the redundant stimulus; the males averaged 37 and 12 leverpresses, respectively. By Sequence 4, the females averaged 15 and 3.5 leverpresses to the original and redundant stimuli, and the males averaged 11 and 3.5 leverpresses, respectively. There were no significant differences in the response rates to the $\mathrm{L}$ (or $\mathrm{T}$ ) whether it was the original or the redundant stimulus. However, the response rate to the original stimulus was consistently higher for females than for males. This was unexpected, because the males were superior in performance during Stages 1 and 2. Although we are unsure of the reason for these findings, another characteristic of conditioning is that animals that have a higher level of conditioning will frequently extinguish more rapidly. This may partially explain these results.

\section{DISCUSSION}

These findings demonstrate that the basic paradigm used in this experiment is a sensitive tool for measuring the sequential development of the blocking phenomenon during Stages 1,2 , and 3 . Because all leverpresses during the stimulus period are rewarded, the paradigm ensures good stimulus control of the leverpress. Thus, the individual importance and interaction of different variables can be readily manipulated and studied in future experiments.

Finally, the nature of the reinforcer must be considered. The literature indicates that aversive reinforcers are usually very effective in their ability to associate stimuli (classical conditioning) or to associate a stimulus and a response (instrumental conditioning). Because these aversive reinforcers are so powerful, however, the ceiling effect is quickly reached and potential subtleties in conditioning may be obscured. This may retard the understanding of how animals are able to function and learn in an environment where electrical shock is not a common reinforcer. We are not suggesting that aversive reinforcers generate erroneous data, but rather that appetitive reinforcers may provide a potentially broader range of data. Although a great deal of research has been done on Mackintosh's (1975) concept of alpha as a learning rate parameter for each specific stimulus, little research has been done on beta as a learning rate parameter (Dickinson \& Mackintosh, 1979; Neely \& Wagner, 1974) for each specific reinforcer. Just as a weak stimulus or a stimulus from a different analyzer would have an alpha different from that of a strong stimulus, an appetitive reinforcer would have a beta value different from that of an aversive reinforcer. The value of beta would differ among different appetitive reinforcers and different aversive reinforcers. The present study was not designed to delineate the rules and conditions that govern beta, but the data do suggest some areas of future research. The use of other reinforcers, such as water for thirsty animals, should yield similar results for blocking, but the level of blocking, rate of acquisition, and extinction rates may differ somewhat from those observed when a food reward is used. Water would thus have a beta different from that of food or electric shock, but the phenomenon of blocking would be valid for all reinforcers.

\section{REFERENCES}

Dickinson, A., \& Mackintosh, N. J. (1979). Reinforcer specificity in the enhancement of conditioning by posttrial surprise. Journal of Experimental Psychology: Animal Behavior Processes, 5, 162-177.

Holland, P. C. (1977). Conditioned stimulus as a determinant of the form of the Pavlovian conditioned response. Journal of Experimental Psychology: Animal Behavior Processes, 3, 77-104.

KAMIN, L. J. (1969). Predictability, surprise, attention and conditioning. In B. A. Campbell \& R. M. Church (Eds.), Punishment and aversive behavior: A symposium (pp. 279-298). New York: AppletonCentury-Crofts.

KolstoE, R. H. (1973). Introduction to statistics for the behavioral sciences (rev. ed.). Homewood, IL: Dorsey.

MaCKINTOSH, N. J. (1975). A theory of attention: Variations in the associability of stimuli with reinforcement. Psychological Review, 82, 276-298.

NeELY, J. H., \& WAGNER, A. R. (1974). Attenuation of blocking with shifts in reward: The involvement of schedule-generated contextual cues. Journal of Experimental Psychology, 102, 751-763.

Rescorla, R. A., \& WAGNer, A. R. (1972). A theory of Pavlovian conditioning: Variations in the effectiveness of reinforcement and nonreinforcement. In A. Black \& W. F. Prokasy (Eds.), Classical conditioning II: Current research and theory. New York: AppletonCentury-Crofts.

\section{NOTE}

1. Mention of a trademark or proprietary product does not constitute a guarantee or warranty of the product by the U.S. Department of Agriculture, and does not imply its approval to the exclusion of other products that may also be suitable.

(Manuscript received for publication October 9, 1986.) 